\title{
A Comparison of Residual Shunts After Two Different Surgical Methods for Isolated Ventricular Septal Defect Closure
}

Hongbo Li

Chongqing Medical University Affiliated Children's Hospital

Chun Wu

Chongqing Medical University Affiliated Children's Hospital

Zhengxia Pan

Chongqing Medical University Affiliated Children's Hospital

Linyun Xi ( $\nabla 1010159025 @ q q . c o m)$

Chongqing Medical University Affiliated Children's Hospital

Research article

Keywords: Residual shunts, Ventricular septal defect, Surgery, Spontaneous closure

Posted Date: October 18th, 2021

DOI: https://doi.org/10.21203/rs.3.rs-966665/v1

License: (c) (i) This work is licensed under a Creative Commons Attribution 4.0 International License.

Read Full License 


\section{Abstract}

\section{Background}

To examine residual defects(RS) and examined the rate of spontaneous RS closure over time to identify factors associated with the occurrence and spontaneous closure of these defects after two different operation methods.

\section{Methods}

In this retrospective analysis, we enrolled only patients with perimembranous VSDs (pmVSDs) and reviewed the clinical records of patients who underwent repair for pmVSDs between January 2016 and January 2019. All patients underwent surgery with either cardiopulmonary bypass (the CPB group) or minimally invasive closure of transthoracic VSDs under the guidance of TEE (the MIC group). There were 189 patients who underwent CPB surgery and 211 patients who underwent MIC surgery. Ultimately, there were 37 patients with RSs in the CPB group (surgical repair via CPB) and 39 patients with RSs in the MIC group (minimally invasive closure of transthoracic VSDs). Postoperatively, all surgical patients were required to return for outpatient follow-up visits, and echocardiography was required to evaluate the RS. Assessments included shunt velocity and RS size.

\section{Results}

In the CPB group, 16 patients had a small RS, and 21 patients had a moderate RS. The comparison between the variables such as weight, preoperative VSD size, and RS size revealed no significant differences except RS size $(P=0.000)$. To compare the spontaneous closure rates of the two sizes of RSs(the moderate group and small size group), Kaplan-Meier plots were used. These plots show that the small size was more likely to undergo spontaneous closure $(P=0.034)$, but the final spontaneous closure rate was not significantly different $(P=1.000)$.In the MIC group, there were 29 patients in the small group and 10 patients in the moderate group, and the variables such as weight, preoperative VSD size, and preoperative VSD size showed no significant differences except RS size and the rate of no spontaneous closure ( $P=0.000$ vs. 0.045). The Kaplan-Meier plots (Figure 2 ) showed that the small size was RSs were more likely to undergo spontaneous closure $(P=0.004)$, while the final spontaneous closure rate was significantly different between groups $(P=0.045)$. At 2 years post-operation, 7 patients still had $R S$, and the overall spontaneous closure rate was $90.8 \%$. The size of the RS on discharge was the only variable identified, on Cox regression, to be predictive of the likelihood of spontaneous closure. The univariate analysis, however, showed that shunt velocity had no association with spontaneous closure. Factors including age, weight, sex, surgical technique, and VSD size had no association with spontaneous closure.

\section{Conclusion}

The RS incidence and spontaneous closure rates were not significantly different two different operation methods. Small RSs were more likely to undergo spontaneous closure in both groups. 


\section{Background}

Ventricular septal defects (VSDs) are among the most common congenital cardiac defects[1]. Surgical closure of VSDs includes conventional surgery with cardiopulmonary bypass (CPB) and minimally invasive closure of transthoracic VSDs under the guidance of transesophageal echocardiography (TEE) [2]. In recent years, minimally invasive closure of transthoracic VSDs has been widely used in China. The results of both surgical approaches are excellent[1-3], but they both have their own advantages and disadvantages[4] [5]. Residual defects, which serve as an important criterion for surgical evaluation, are frustrating complications that are frequently observed on intraoperative TEE or postoperative transthoracic echocardiography (TTE), but the spontaneous closure rates and outcomes of persistent residual shunts (RSs) remain to be clearly defined. Few studies have reported persistent RSs, especially in comparisons of these two different surgical strategies. We evaluated our results regarding postoperation residual defects and examined the rate of spontaneous RS closure over time to identify factors associated with the occurrence and spontaneous closure of these defects. To the best of our knowledge, this is the first study comparing RSs between two surgical methods.

\section{Materials And Methods}

\section{Patient population}

In this retrospective analysis, we enrolled only patients with perimembranous VSDs (pmVSDs) and reviewed the clinical records of patients who underwent repair for pmVSDs between January 2016 and January 2019. All patients underwent surgery with either cardiopulmonary bypass (the CPB group) or minimally invasive closure of transthoracic VSDs under the guidance of TEE (the MIC group). Of the two groups confirmed to have received an intervention at our centre, only patients who met the following criteria were included in study: 1 . isolated pmVSD with no other intracardiac malformation, significant left-to-right shunt, and ventricular overload with or without pulmonary hypertension; 2 . age older than 6 months; 3 . weight more than $10 \mathrm{~kg}$; and 4 . VSD size was no more than $8 \mathrm{~mm}$. The exclusion criteria were as follows: respiratory diseases, history of a thoracic procedure, severe valvular regurgitation, and right-toleft shunt caused by severe pulmonary hypertension.

There were 189 patients who underwent CPB surgery and 211 patients who underwent MIC surgery. Ultimately, there were 37 patients with RSs in the CPB group (surgical repair via CPB) and 39 patients with RSs in the MIC group (minimally invasive closure of transthoracic VSDs). The clinical data of all patients with RSs are shown in Table 1. 
Table 1

Demographics and clinical characteristics of the two groups

\begin{tabular}{|c|c|c|c|}
\hline & CPB group & MIC group & $P$ \\
\hline$N$ & 37 & 39 & \\
\hline the RS incidence rate & $19.6 \%(37 / 189)$ & $18.5(39 / 211)$ & 0.781 \\
\hline 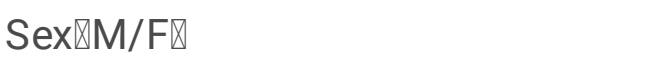 & $21 / 16$ & $17 / 22$ & 0.359 \\
\hline Age $(m)$ & $36.6 \pm 23.0$ & $27.9 \pm 20.4$ & 0.085 \\
\hline Weight (kg) & $13.9 \pm 3.4$ & $12.6 \pm 3.2$ & 0.072 \\
\hline VSD size $(\mathrm{mm})$ & $6.7 \pm 2.4$ & $6.1 \pm 1.3$ & 0.219 \\
\hline Small RS/Moderate RS & $16 / 21$ & $29 / 10$ & 0.01 \\
\hline RS size $(\mathrm{mm})$ & $1.9 \pm 0.48$ & $1.7 \pm 0.38$ & 0.115 \\
\hline Postoperation shunt velocity $(\mathrm{m} / \mathrm{s})$ & $3.00 \pm 0.74$ & $2.65 \pm 0.513$ & 0.016 \\
\hline
\end{tabular}

A residual shunt was defined when colour Doppler flow mapping showed a shunt across the interventricular septum along the margins of the patch or device occlusion. One paper classified the RSs as trivial ( $<1 \mathrm{~mm}$ colour jet width), small (1-2 mm colour jet width), or moderate (2-4 mm colour jet width)[6], and other papers classified the RSs into two groups (RSs smaller and larger than $1.25 \mathrm{~mm}$ )[7]; in our case series, RSs were graded as small $(<2 \mathrm{~mm})$ or moderate $(>2 \mathrm{~mm})$.

The end point was defined as spontaneous closure of the RS, as evaluated by echocardiography, within two years post-operation.

\section{Surgical technique}

In the CPB group, conventional surgical repair was conducted via a median sternotomy approach under CPB. A Gore-tex patch was used in all patients.

In the MIC group, periventricular device closure was performed, which has been described previously[8]. Perioperatively, TEE was used to assess the size of the VSD, and the movement of the aortic valve and tricuspid valve was very important. A minimal incision (approximately $3 \mathrm{~cm}$ ) was made through a lower inferior median sternotomy. The pericardium was cradled, and the right ventricle was exposed. An appropriate puncture point was located with tweezers under the guidance of TEE. The guidewire was slowly advanced through the VSD into the left ventricle, the dilator was retreated, and the selected delivery sheath was introduced through the guidewire into the left ventricle to establish a delivery pathway. The wire and the inner dilator were removed. The occluder was loaded into the delivery sheath with the help of a loading cable. Then, the occluder was advanced to the tip of the sheath, and the left disc was deployed. The sheath was pulled back slowly until the left disc approximated the ventricular septum and the right 
disc was deployed in turn. During deployment of the asymmetric device, the occluder was gently rotated to ensure that the platinum marker of the distal disk pointed to the apex of the heart and could thus avoid interaction with the aortic valve.

\section{Long-term follow-up}

Postoperatively, all surgical patients were required to return for outpatient follow-up visits, and echocardiography was required to evaluate the RS. Assessments included shunt velocity and RS size. All patients were required to visit our hospitals for routine examinations.

\section{Statistical analysis}

Quantitative data are expressed as the mean \pm standard deviation as appropriate. All P values were twosided, and $\mathrm{P}<0.05$ was considered to indicate statistical significance. Age, sex, and type were analysed by using the chi-square test. Quantitative data were analysed by using a t test. Survival data are presented by means of the Kaplan-Meier method. Stepwise Cox regression was used to identify factors associated with spontaneous closure.

\section{Results}

In the MIC group, there were 17 males and 22 females. The median weight at surgery was $12.6 \pm 3.2 \mathrm{~kg}$, the median age at surgery was $27.9 \pm 20.4$ months, the preoperative VSD size was $6.1 \pm 1.3 \mathrm{~mm}$, the postoperative RS size was $1.7 \pm 0.38 \mathrm{~mm}$, and the postoperative shunt velocity was $2.65 \pm 0.513 \mathrm{~m} / \mathrm{s}$. There were 37 patients in the CPB group, of whom 21 were male and 16 were female. The median weight at surgery was $13.9 \pm 3.4 \mathrm{~kg}$, the median age at surgery was $36.6 \pm 23.0$ months, the preoperative VSD size was $6.7 \pm 2.4 \mathrm{~mm}$, the postoperative RS size was $1.9 \pm 0.48 \mathrm{~mm}$, and the postoperative shunt velocity was $3.00 \pm 0.74 \mathrm{~m} / \mathrm{s}$. The comparison between the variables revealed no significant differences. The patient demographics are shown in Table 1.

In the CPB group, 16 patients had a small RS, and 21 patients had a moderate RS. Variables such as weight, preoperative VSD size, and RS size are listed in Table 2. The comparison between the variables revealed no significant differences except RS size $(P=0.000)$. In the small size group, one patient still had a $1.4 \mathrm{~mm}$ RS at 2 years post-operation, and the size of the RS was $1.8 \mathrm{~mm}$ before hospital discharge. In the moderate group, 2 patients had RSs at 2 years post-operation, with sizes of $1.7 \mathrm{~mm}$ and $2.1 \mathrm{~mm}$, and the sizes of the RSs before hospital discharge were $2.4 \mathrm{~mm}$ and $2.7 \mathrm{~mm}$, respectively. To compare the spontaneous closure rates of the two sizes of RSs(the moderate group and small size group), KaplanMeier plots were used (Figure 1). These plots show that the small size was more likely to undergo spontaneous closure $(P=0.034)$, but the final spontaneous closure rate was not significantly different $(P=1.000)$. 
Table 2

Comparison of demographic and clinical characteristics between the two groups within the CPB group

\begin{tabular}{|llll|}
\hline & Small size & Moderate size & P \\
\hline $\mathrm{N}$ & 16 & 21 & \\
\hline Sex(M/F) & $9 / 7$ & $12 / 9$ & 1.000 \\
\hline Age $(\mathrm{m})$ & $31.2 \pm 17.2$ & $40.8 \pm 26.2$ & 0.212 \\
\hline Weight $(\mathrm{kg})$ & $13.4 \pm 3.4$ & $14.3 \pm 3.4$ & 0.435 \\
\hline VSD size $(\mathrm{mm})$ & $7.1 \pm 2.3$ & $6.4 \pm 2.5$ & 0.403 \\
\hline RS size $(\mathrm{mm})$ & $1.5 \pm 0.25$ & $2.34 \pm 0.18$ & 0.000 \\
\hline No spontaneous closure/spontaneous closure $(\mathrm{N})$ & $1 / 15$ & $2 / 19$ & 1.000 \\
\hline Postoperation shunt velocity $(\mathrm{m} / \mathrm{s})$ & $2.98 \pm 0.64$ & $3.03 \pm 0.82$ & 0.863 \\
\hline
\end{tabular}

Table 3

Comparison of demographic and clinical characteristics between the two groups within the MIC group

\begin{tabular}{|llll|}
\hline & Small size & Moderate size & $\mathrm{P}$ \\
\hline $\mathrm{N}$ & 29 & 10 & \\
\hline Sex\M/F囚 & $12 / 17$ & $5 / 5$ & 0.721 \\
\hline Age $(\mathrm{m})$ & $29.41 \pm 22.2$ & $23.7 \pm 13.6$ & 0.451 \\
\hline Weight $(\mathrm{kg})$ & $12.7 \pm 3.56$ & $12.2 \pm 2.01$ & 0.684 \\
\hline VSD size $(\mathrm{mm})$ & $6.04 \pm 1.36$ & $6.45 \pm 1.11$ & 0.400 \\
\hline RS size $(\mathrm{mm})$ & $1.56 \pm 0.26$ & $2.24 \pm 0.08$ & 0.000 \\
\hline No spontaneous closure/spontaneous closure $(\mathrm{N})$ & $1 / 28$ & $3 / 7$ & 0.045 \\
\hline Postoperative shunt velocity $(\mathrm{m} / \mathrm{s})$ & $2.55 \pm 0.45$ & $2.92 \pm 0.60$ & 0.095 \\
\hline
\end{tabular}

In the MIC group, there were 29 patients in the small group and 10 patients in the moderate group, and the variables such as weight, preoperative VSD size, and preoperative VSD size showed no significant differences except RS size and the rate of no spontaneous closure ( $P=0.000$ vs. 0.045 ). In the small group, one patient still had a $1.3 \mathrm{~mm}$ RS at 2 years post-operation, and the size of the RS was $1.9 \mathrm{~mm}$ before hospital discharge. In addition, in the moderate group, there were 3 patients with RS sizes of 2.2 $\mathrm{mm}, 2.3 \mathrm{~mm}$, and $2.3 \mathrm{~mm}$, respectively, before hospital discharge, and the RSs had not undergone 
spontaneous closure at 2 years post-operation, with sizes of $1.7 \mathrm{~mm}$ and $1.3 \mathrm{~mm}$, and $1.5 \mathrm{~mm}$, respectively. The Kaplan-Meier plots (Figure 2) showed that the small size was RSs were more likely to undergo spontaneous closure $(P=0.004)$, while the final spontaneous closure rate was significantly different between groups $(P=0.045)$.

In the CPB group, 13 patients still had RSs at 1 year post-operation, with a rate of spontaneous closure of $64.8 \%$, and at 2 years post-operation, only 3 patients still had RSs, with a rate of spontaneous closure of $91.9 \%$. In the MIC group, the spontaneous closure rate was $74.4 \%$ at 1 year post-operation, 10 patients still had RSs, and the spontaneous closure rate was $89.7 \%$ at 2 years post-operation, 4 patients who still had RSs; these patients were more likely to have a small RS than those in the CPB group $(P=0.010)$. The Kaplan-Meier plots (Figure 3 ) showed that the time to spontaneous closure in the CPB group and MIC group was not significantly different $(P=0.654)$.

At 2 years post-operation, 7 patients still had RSs, and the overall spontaneous closure rate was $90.8 \%$. The size of the RS on discharge was the only variable identified, on Cox regression, to be predictive of the likelihood of spontaneous closure. The univariate analysis, however, showed that shunt velocity had no association with spontaneous closure (Table 5). Factors including age, weight, sex, surgical technique, and VSD size had no association with spontaneous closure.

Table 4

Comparison of demographic and clinical characteristics between the CPB group and MIC group

\begin{tabular}{|llll|}
\hline & CPB group & MIC group & P \\
\hline N & 37 & 39 & \\
\hline Sex (M/F) & $21 / 16$ & $17 / 22$ & \\
\hline Age (m) & $36.6 \pm 23.0$ & $27.9 \pm 20.4$ & 0.085 \\
\hline Weight $(\mathrm{kg})$ & $13.9 \pm 3.4$ & $12.6 \pm 3.2$ & 0.072 \\
\hline VSD size (mm) & $6.7 \pm 2.4$ & $6.1 \pm 1.3$ & 0.219 \\
RS size (mm) & $1.9 \pm 0.48$ & $1.7 \pm 0.38$ & 0.115 \\
\hline Postoperative shunt velocity (m/s) & $3.00 \pm 0.74$ & $2.65 \pm 0.513$ & 0.016 \\
\hline Spontaneous closure rate (1 year, \%) & 64.8 & 74.4 & 0.456 \\
\hline Spontaneous closure rate (2 years, \%) & 91.9 & 89.7 & 1.000 \\
\hline Small RS/Moderate RS (N) & $16 / 21$ & $29 / 10$ & 0.010 \\
\hline Spontaneous closure/No spontaneous closure (N) & $34 / 3$ & $35 / 4$ & 1.000 \\
\hline
\end{tabular}


Table 5

Cox regression for spontaneous closure

\begin{tabular}{|lllllll|}
\hline Variable & \multicolumn{2}{l}{ Univariate } & \multicolumn{3}{l|}{ Multivariate } \\
\cline { 2 - 5 } & HR & $95 \% \mathbf{C l}$ & P value & HR & $95 \%$ Cl & P value \\
\hline Age & 0.993 & $(0.979,1.008)$ & 0.363 & & & \\
\hline Weight & 1.019 & $(0.920,1.128)$ & 0.723 & & & \\
\hline Sex & 1.286 & $(0.761,2.173)$ & 0.247 & & & \\
\hline Technique & 1.253 & $(0.724,2.169)$ & 0.419 & & & \\
\hline VSD size & 0.927 & $(0.804,1.070)$ & 0.302 & & & \\
\hline RS size & 0.229 & $(0.118,0.443)$ & 0.000 & 0.241 & $(0.127,0.459)$ & 0.000 \\
\hline Shunt velocity & 0.981 & $(0.683,1.408)$ & 0.916 & & & \\
\hline
\end{tabular}

\section{Discussion}

Surgical closure of VSDs includes conventional surgery with CPB and minimally invasive closure of transthoracic VSDs under the guidance of TEE. Using standardized surgical techniques and postoperative care management, the outcomes of both surgeries are excellent, with mortality and morbidity rates approaching zero in almost all centres internationally[9]. However, as one of the major complications, RSs are not as rare as we would like to believe in these two surgeries.

RSs, which are an important indicator for the surgical treatment of VSDs, have attracted increasing attention. The incidence of RSs after CPB reported by Deng was 31.2\%[7], and an incidence of 35\% was reported by Dodge-Khatami[9]. The incidence of RSs after transthoracic device closure of VSDs was $15.8 \%$, as reported by Ren C[8]. In our series, the rates of RSs after CPB and periventricular device closure were $19.6 \%$ and $18.4 \%$, respectively, which were similar to other reports.

According to the relevant literature and our experience, the reasons for postoperative residual shunts in the CPB group are as follows. First, during the operation, the margin of the VSD was not clearly exposed, and the suture was not tight and formed a gap. Second, the suture at the corner of the VSD was not well gap uniformity. Third, the patch was not perfectly clipped, and a wrinkle was formed. Fourth, the suture was shallow, the myocardium was avulsed, and an RS formed. In the MIC group, the main factor associated with RSs was whether the shape of the occluder fit the VSD. Most of the time, the margin of the VSD was bumpy and irregular, even though the tricuspid chordae tendineae was crossed, which would prevent the occluder from covering the entire VSD.

The Kaplan-Meier plots (Figure 1) showed that in the CPB group, for those patients with small RSs, the curve slumped faster than that of those with moderate RSs $(P=0.034)$, but the groups tended to reach an approximate closure rate 2 years after discharge, which means that small RSs were more likely to 
undergo spontaneous closure, so there were no differences in the rate of spontaneous closure at the end of the follow-up $(P=1.000)$. Another Kaplan-Meier plot (Figure 2) showed the same result in the MIC group, but one difference compared with the CPB group was that the moderate RS group tended to have difficulty undergoing spontaneous closure $(p=0.045)$, which was hard to explain. In both groups, we found that factors including age, weight, sex, and VSD size showed no significant differences between small- and moderate-sized RSs, which indicates that RS closure was not connected to the above factors. An interesting finding of our study was that more small RSs occurred in the MIC group than in the CPB group because the occluder could be adjusted under the guidance of TEE (Table 4). As shown in Table 4, we found that factors including age, weight, sex, and VSD size were not significantly different between the two groups, which indicates that the size of the RS generated in the operation was not connected to the above factors. We also found that the spontaneous closure rate at 1 year and 2 years post-operation were not significantly different between the two groups, and the spontaneous closure rate at 1 year postoperation was approximately $70 \%$ in both groups and approximately $90 \%$ at two years (Figure 3 ).

The mechanism of VSD spontaneous closure is still not clear and may vary with the site of the defect, although many hypotheses have been proposed. Roberts[10] speculated that closure of the RS resulted from endocardial proliferation, probably stimulated by turbulent blood flow through the RS, and RS would not closure without the superimposition of an active inflammatory process. Anderson[11] suggested that spontaneous closure involved an adherent tricuspid valve leaflet, tricuspid leaflet tissue tags, subaortic tissue tags, prolapsed aortic valve leaflets, or a combination of these factors. Takaki et al[12] demonstrated that the jet stream could create a tricuspid pouch, which was formed by the left-to-right shunt flow jet towards the tricuspid valve, followed by deposition of fibrin over the margins of the defect and adhesion of the septal leaflet of the tricuspid valve to the margin of the VSD.

We performed a Cox regression analysis to identify factors associated with spontaneous closure. The only variable we found to be significant was the size of the RS on initial postoperative echocardiography, which is supported by findings from other groups[9]. Age, weight, sex, surgical technique, preoperative VSD size and shunt velocity were not significant factors. Although shunt velocity was reported[13] to be a significant factor in RS spontaneous closure, it was not possible to draw this conclusion from our data. Some reports also reached this conclusion. Some reports[14, 15] proposed that age has a significant influence on the incidence of spontaneous VSD closure, while in our series, because of our criteria, the age span may not be large enough. Additional research should include patients of different ages, especially young patients.

In terms of limitations, this study was a retrospective review with all the inherent deficiencies of such studies. This was a retrospective single-centre study, and the sample size was small; thus, the results may not be applicable to the population as a whole. The type of VSD was limited to perimembranous VSDs, and the age span was not large enough.

In conclusion, similar to other procedures, MIC of transthoracic VSDs can also lead to RSs. We compared conventional surgery with $\mathrm{CPB}$ and MIC and found that the RS incidence and spontaneous closure rates 
were not significantly different. Small RSs were more likely to undergo spontaneous closure in both groups.

\section{List Of Abbreviations}

residual shunts (RSs); Ventricular septal defects (VSDs); cardiopulmonary bypass (CPB); transesophageal echocardiography (TEE); perimembranous VSDs (pmVSDs)

\section{Declarations}

Ethics approval and consent to participate: This research received ethical approval and consent.

Consent for publication: All patient information contained in this manuscript has been published with the consent of their parents.

Availability of data and materials: The data and materials in the manuscript are available, and the original data for the relevant results are owned by myself; I can be contacted if needed.

Competing interests: There are no conflicts of interest or legal economic disputes in this study.

Funding: Not applicable.

Authors' contributions: Hongbo Li, the first author, designed the work, acquired, analyzed, and interpreted the data, drafted the work and substantively revised it. Chun Wu, the second author, substantively revised it. Zhengxia Pan, the third author, provided some suggestions for this work. Linyun Xi, the corresponding author, designed the work, drafted the work, substantively revised it, and approved the submitted version.

Acknowledgements: I would like to thank all the doctors and nurses of the Cardiothoracic Surgery Department of the Children's Hospital of Chongqing Medical University for giving me so much advice and help. I appreciate my family for their companionship, support and care.

\section{References}

1. Penny D, Vick GW: Ventricular septal defect. Lancet 2011, 377(9771):1103-1112.

2. Anderson BR, Stevens KN, Nicolson SC, Gruber SB, Spray TL, Wernovsky G, Gruber PJ: Contemporary outcomes of surgical ventricular septal defect closure. Journal of Thoracic \& Cardiovascular Surgery 2013, 145(3):641-647.

3. Schipper M, Slieker MG, Schoof PH, Breur J: Surgical Repair of Ventricular Septal Defect; Contemporary Results and Risk Factors for a Complicated Course. Pediatric Cardiology 2017, 38(2):264-270.

4. Xing Q, Pan S, Qi A, Zhang Z, Li J, Feng L, Qin W, Zhuang Z: Minimally invasive perventricular device closure of perimembranous ventricular septal defect without cardiopulmonary bypass: Multicenter 
experience and mid-term follow-up. The Journal of Thoracic and Cardiovascular Surgery 2010, 139(6):1409-1415.

5. Roos-Hesselink JW, Meijboom FJ, Spitaels S, Domburg RV, Simoons ML: Outcome of patients after surgical closure of ventricular septal defect at young age: Longitudinal follow-up of 22-34 years. European Heart Journal 2004, 25(12):1057-1062.

6. Oses P, Hugues N, Dahdah N, Vobecky SJ, Miro J, Pellerin M, Poirier NC: Treatment of isolated ventricular septal defects in children: Amplatzer versus surgical closure. Annals of Thoracic Surgery 2010, 90(5):1593-1598.

7. Deng $X$, Huang P, Luo J, Chen R, He C: Residual Shunts Following Isolated Surgical Ventricular Septal Defect Closure: Risk Factors and Spontaneous Closure. Pediatric Cardiology 2020, 41(1).

8. Ren C, Wu C, Pan Z, Li Y: Minimally invasive closure of transthoracic ventricular septal defect: postoperative complications and risk factors. Journal of Cardiothoracic Surgery 2021, 16(1).

9. Dodge-Khatami A, Knirsch W, Tomaske M, Prêtre R, Bettex D, Rousson V, Bauersfeld U: Spontaneous Closure of Small Residual Ventricular Septal Defects After Surgical Repair. Annals of Thoracic Surgery 2007, 83(3):902-905.

10. Roberts WC, Morrow AG, Mason DT, Braunwald E: Spontaneous closure of ventricular septal defect, anatomic proof in an adult with tricuspid atresia. Circulation 1963, 27(1):90.

11. Anderson RH, Lenox CC, Zuberbuhler JR: Mechanisms of closure of perimembranous ventricular septal defect. Am J Cardiol 1983, 52(3):341-345.

12. Takaki A, Ogawa H, Wakeyama T, Iwami T, Kimura M, Uchinoumi H, Akashi S, Matsuda S, Miyazaki Y, Matsuzaki M: Tricuspid Pouch can Cause Systemic Embolization in Adulthood. Circulation Journal Official Journal of the Japanese Circulation Society 2006, 70(5):631.

13. Glancy DL, Roberts WC: Complete spontaneous closure of ventricular septal defect : Necropsy study of five subjects. American Journal of Medicine 1967, 43(6):846-853.

14. Zhang J, Ko JM, Guileyardo JM, Roberts WC: A review of spontaneous closure of ventricular septal defect. Baylor University Medical Center Proceedings 2017, 28(4):516-520.

15. Yang $X$, Yang S: Factors influencing the spontaneous closure of ventricular septal defect in infants. Journal of the American College of Cardiology 2014, 64(Supplement):C192-C193.

\section{Figures}




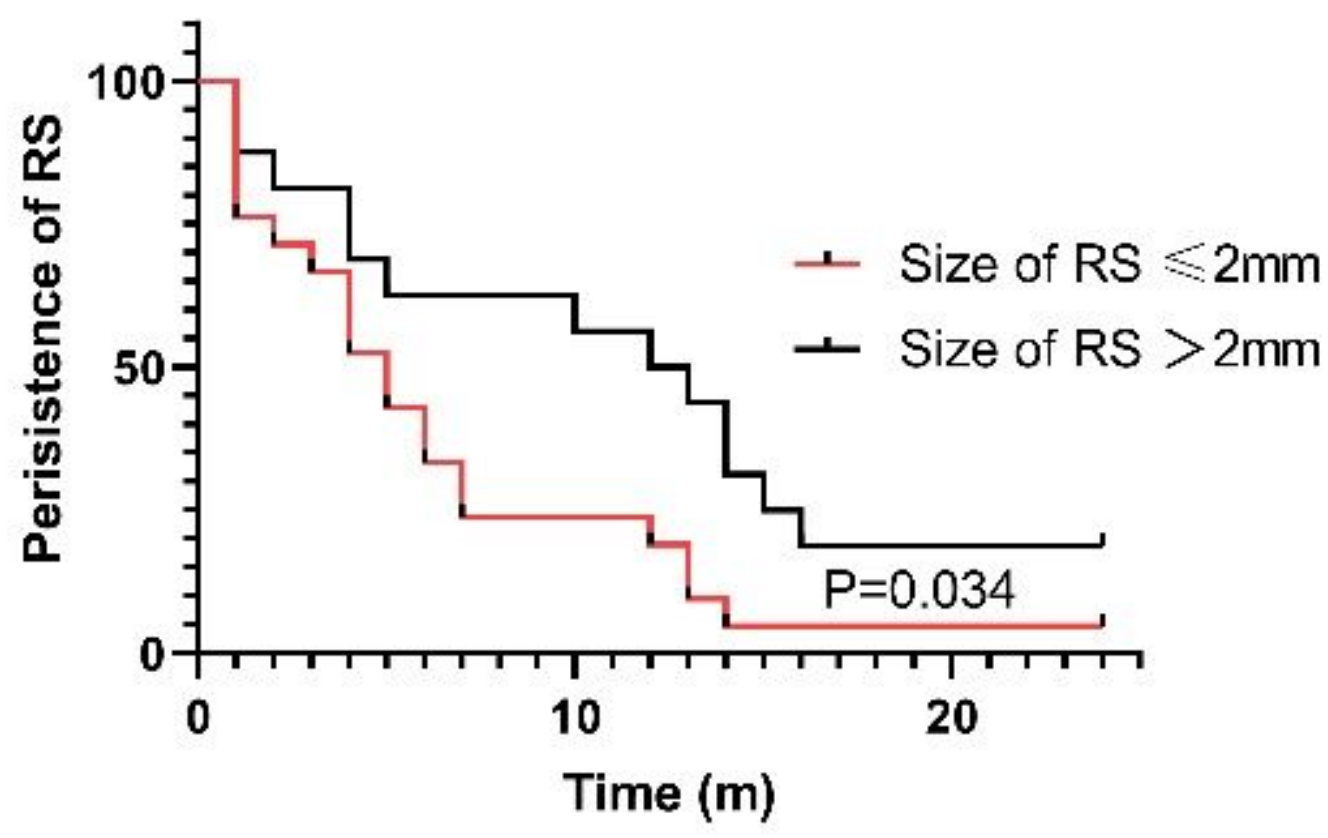

Figure 1

Kaplan-Meier plot showing different spontaneous closure rates for $\mathrm{RS} \leq 2 \mathrm{~mm}$ and $\mathrm{RS}>2 \mathrm{~mm}$ in the CPB group

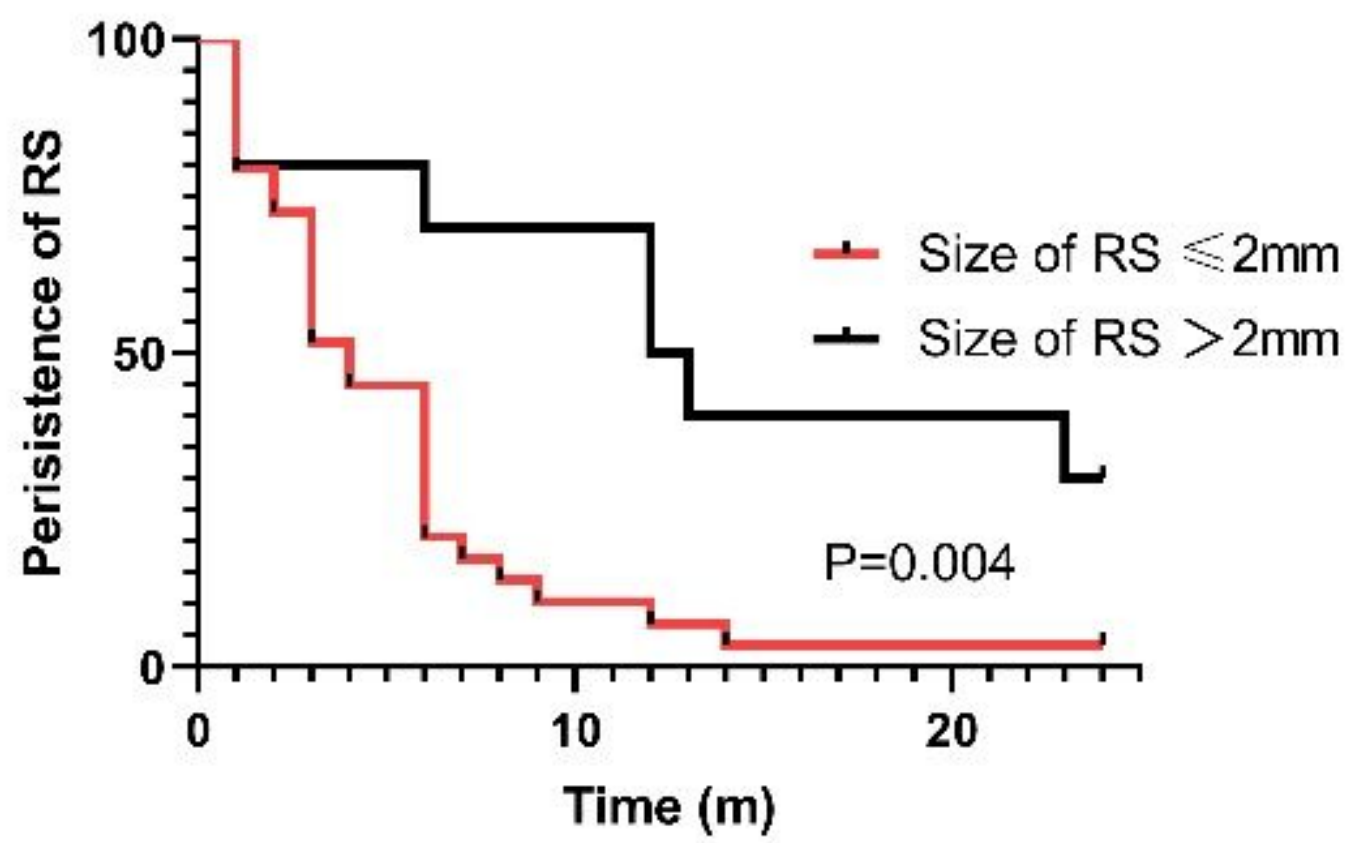

Figure 2

Kaplan-Meier plot showing different spontaneous closure rates for $\mathrm{RS} \leq 2 \mathrm{~mm}$ and $\mathrm{RS}>2 \mathrm{~mm}$ in the MIC group 


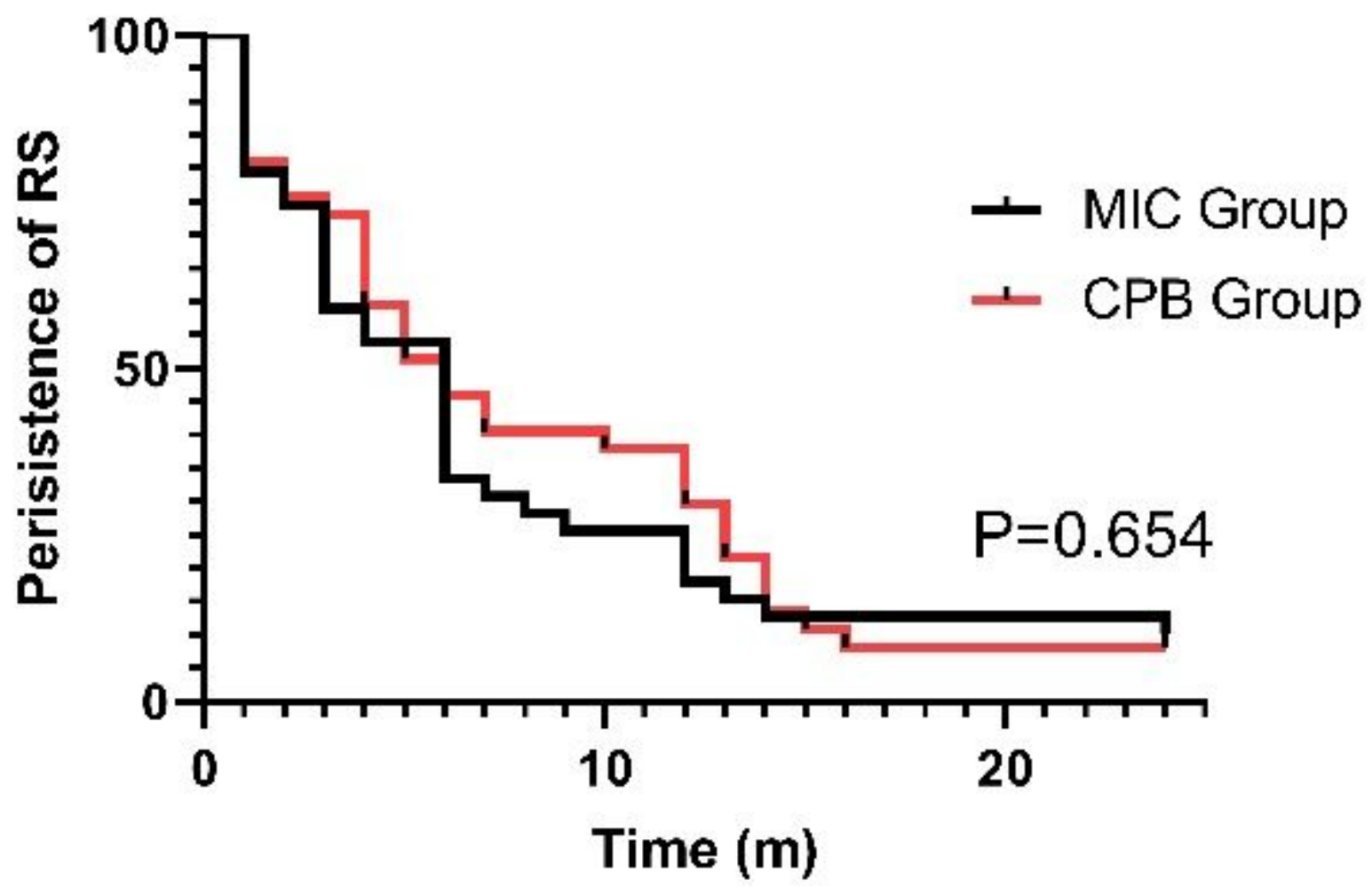

Figure 3

Kaplan-Meier plot showing no difference in the spontaneous closure rate between the MIC group and the CPB group. 